\title{
Effect of Partially Screened Nuclei on Fast-Electron Dynamics
}

\author{
L. Hesslow, ${ }^{1, *}$ O. Embréus, ${ }^{1}$ A. Stahl, ${ }^{1}$ T. C. DuBois, ${ }^{1}$ G. Papp, ${ }^{2}$ S. L. Newton, ${ }^{1}$ and T. Fülöp ${ }^{1}$ \\ ${ }^{1}$ Department of Physics, Chalmers University of Technology, 41296 Gothenburg, Sweden \\ ${ }^{2}$ Max-Planck-Institute for Plasma Physics, D-85748 Garching, Germany
}

(Received 31 January 2017; published 20 June 2017)

\begin{abstract}
We analyze the dynamics of fast electrons in plasmas containing partially ionized impurity atoms, where the screening effect of bound electrons must be included. We derive analytical expressions for the deflection and slowing-down frequencies, and show that they are increased significantly compared to the results obtained with complete screening, already at subrelativistic electron energies. Furthermore, we show that the modifications to the deflection and slowing down frequencies are of equal importance in describing the runaway current evolution. Our results greatly affect fast-electron dynamics and have important implications, e.g., for the efficacy of mitigation strategies for runaway electrons in tokamak devices, and energy loss during relativistic breakdown in atmospheric discharges.
\end{abstract}

DOI: 10.1103/PhysRevLett.118.255001

Introduction.-Fast electrons, having speeds well above the thermal speed of the bulk plasma population, are ubiquitous in space and laboratory plasmas. An important process leading to such high-energy electrons is the runaway mechanism. Runaway electrons can be produced in the presence of an accelerating electric field if it exceeds the critical value $E_{c}=n_{e} e^{3} \ln \Lambda_{0} / 4 \pi \epsilon_{0}^{2} m_{e} c^{2}$ [1,2]. Situations where runaway electrons are believed to be important include solar flares [3], atmospheric discharges [4,5], laser-produced plasmas [6], as well as tokamak disruptions [7]. In the latter, it is important to understand the dynamics of runaway electrons as they have the potential to seriously damage the tokamak [8]. This will be especially problematic in larger, future tokamak experiments, such as ITER [9]. There, runawayelectron currents in excess of a megaampere are expected to form if a disruption is not mitigated, and the potential damage associated with such currents is larger than in any present experiment [10]. Therefore, reliable methods to deal with such currents are required [11,12].

One method to mitigate the detrimental effects of runaway-electron beams is to dissipate them by injecting heavy ions (impurities) such as argon or neon. The impurity atoms become weakly ionized in the cold (few eV) postdisruption plasma, and act to collisionally scatter particles in the high-energy electron beam. Experiments have shown that such injection of impurities with high atomic mass can shift the energy distribution of the fast electrons towards lower energies, to a much larger extent than predicted by standard collisional theory $[10,13]$. The discrepancy between measured and predicted dissipation increases with atomic mass, even though the ions are usually only singly ionized in the cold, post-disruption phase. This is an indication that a fast electron is not simply deflected by the Coulomb interaction with the net charge of the ion, but also probes its internal electron structure, so that the nuclear charge is not completely screened. The fast electrons can therefore be expected to experience higher collision rates against impurities, leading to a more efficient damping, in agreement with experimental observations.

To quantify the importance of this partial penetration of the electron cloud of the ion, we compare the low-momentumtransfer limit of complete screening (CS) of the nuclear charge (i.e., the electron interacts only with the net ion charge) to the high-energy limit of no screening (the electron experiences the full nuclear charge), for both elastic and inelastic collisions. For elastic collisions, where the ion can be modeled as one entity [14], screening affects the interaction strength, which is proportional to the charge squared. Compared to the limit of complete screening, the case of no screening thus enhances the interaction strength by a factor $X^{2}=\left(Z / Z_{0}\right)^{2}$, where $Z_{0}$ is the ionization state and $Z$ is the charge number of the nucleus. Furthermore, inelastic collisions (leading to excitation of the ion) can be treated as electron-electron interactions [15], and thus increase the effective electron density of the plasma (as experienced by the fast electron). The rate of electron-electron collisions will therefore be of order $X$ larger, as compared to the case where only free electrons are included. Since the factor $X$ is large when weakly ionized high-atomic-number ions are present in the plasma, the effect of reduced screening on the collisional dynamics of fast particles can be significant for both elastic and inelastic collisions.

To model the reduced-screening effect, a quantummechanical model must be adopted. Because of the high speed of the incoming electrons, the elastic collisions can be treated using the Born approximation [16,17], which requires knowledge of the electronic charge density of the impurity ion. The effect of inelastic collisions can be modeled using Bethe's theory for the collisional stopping power [18]. A classical description of elastic collisions, combined with a stopping-power formula for inelastic collisions [18], was used in a test-particle approach in Ref. [19]. The results indicated that the effect of the partially ionized impurities on the runaway growth rate 
can be substantial. However, without a quantum-mechanical treatment of the elastic collisions and a solution of the kinetic equation for the fast-electron distribution, the effect of screening on fast-electron dynamics cannot be accurately quantified. An extension to a quantum-mechanical treatment of elastic collisions in the Born approximation was employed in Refs. $[4,5,16,17]$ using the Thomas-Fermi theory for the electron charge density, which is limited to intermediate distances from the nucleus, and does not capture the shell structure of the ion [14]. Based on the results of Ref. [16], it was pointed out in Ref. [20] that the runaway generation rate is expected to be reduced. However, an accessible analytical model of screening, that can be incorporated in kinetic simulations of runaway-electron dynamics, has not been derived. This is essential in order to combine all of the important effects governing runaway dynamics into a tractable kinetic model, which would enable a quantification of the effect of reduced screening on the runaway-electron distribution.

In this Letter we present a generalized collision operator which accounts for the screening effect of bound electrons in collisions between fast electrons and partially ionized impurities. We model elastic electron-ion collisions quantum mechanically in the Born approximation, using density functional theory (DFT) to obtain the electron-density distribution of the impurity ions. This allows us to determine the deflection frequency from first principles, without the assumption of infinite nuclear charge used in the Thomas-Fermi model. Furthermore, we employ stopping-power theory to describe inelastic collisions with the bound electrons, and derive an expression for the slowingdown frequency. We demonstrate the effect of screening on the electron distribution function via kinetic simulations.

Collision operator.-Small-angle collisions between species $a$ and $b$ can be described by the Fokker-Planck operator [21,22]: $C^{a b}=-\nabla_{k}\left(f_{a}\left\langle\Delta p^{k}\right\rangle_{a b}\right)+\frac{1}{2} \nabla_{k} \nabla_{l}\left(f_{a}\left\langle\Delta p^{k} \Delta p^{l}\right\rangle_{a b}\right)$, where $f_{a}$ is the distribution function of particle species $a$, $\mathbf{p}=\gamma \mathbf{v} / c$ is the normalized momentum (with $\gamma$ the Lorentz factor), and $\Delta p^{k}$ the change in the $k$ th component of the particle momentum in a collision. The momentum averages are given by $\left\langle\Delta p^{k} \cdots \Delta p^{l}\right\rangle_{a b}=\int d \mathbf{p}^{\prime} f_{b}\left(\mathbf{p}^{\prime}\right) \int\left(d \sigma_{a b} /\right.$ $d \Omega) u \Delta p^{k} \cdots \Delta p^{l} d \Omega$, where $u$ is the relative velocity between the particles, and $d \sigma_{a b} / d \Omega$ is the differential cross section.

When species $b$ has a Maxwellian distribution, the collision operator can be simplified to

$$
C^{a b}=\nu_{D}^{a b} \mathcal{L}\left(f_{a}\right)+\frac{1}{p^{2}} \frac{\partial}{\partial p}\left[p^{3}\left(\nu_{S}^{a b} f_{a}+\frac{1}{2} \nu_{\|}^{a b} p \frac{\partial f_{a}}{\partial p}\right)\right],
$$

where $\mathcal{L}$ represents scattering at constant energy [23], and $\nu_{D}^{a b}, \nu_{S}^{a b}$, and $\nu_{\|}^{a b}$ are the deflection, slowing-down, and parallel-diffusion frequencies which are well known in the limits of complete and no screening [23]. In this Letter, we derive a generalization of these frequencies, taking into account the effect of reduced screening in the elastic collisions between electrons and ions, as well as inelastic collisions between fast and bound electrons.

Elastic collisions.-Elastic collisions with ions contribute to pitch-angle scattering (deflection frequency) through the scattering cross section, which we evaluate in the Born approximation. The Born approximation is valid for $\beta=$ $v / c \gg Z \alpha$ [14], where $\alpha \approx 1 / 137$ is the fine-structure constant. In the cross section, we neglect ion recoil for all ion species $j$ (since $m_{e} / m_{j} \ll 1$ ). Furthermore, since we are interested in the effect on superthermal particles with $v \gg v_{T e} \gg v_{T j}$, where $v_{T a}=\sqrt{2 T_{a} / m_{a}}$ is the thermal speed, we consider collisions with a narrow ion distribution: $f_{j}(\mathbf{p})=n_{j} \delta(\mathbf{p})$. The cross section then takes the following form [14,24]:

$$
\frac{d \sigma_{e j}}{d \Omega}=\frac{r_{0}^{2}}{4 p^{4}}\left(\frac{\cos ^{2}(\theta / 2) p^{2}+1}{\sin ^{4}(\theta / 2)}\right)\left|Z_{j}-F_{j}(q)\right|^{2},
$$

where $r_{0}$ is the classical electron radius, $\theta$ is the deflection angle, and $\mathbf{q}=2 \mathbf{p} \sin (\theta / 2) / \alpha$. The atomic form factor $F_{j}(q)$ is the Fourier transform of the electron number density $\rho_{e, j}$ around an ion of species $j$ :

$$
F_{j}(q)=\int \rho_{e, j}(r) e^{-i \mathbf{q} \cdot r / a_{0}} d \mathbf{r},
$$

where $a_{0}=\hbar /\left(m_{e} c \alpha\right)$ is the Bohr radius. In the limit of small $q$, the form factor approaches the number of bound electrons $N_{e, j}=Z_{j}-Z_{0, j}$, giving a factor of $Z_{0, j}^{2}$ in the cross section in Eq. (2), which corresponds to complete screening. In the opposite limit of high momentum, the fast oscillation of the integrand causes the form factor to vanish, giving a factor of $Z_{j}^{2}$, representing no screening.

When deriving the generalized collision operator using Eqs. (2) and (3), we retain $x=\sin (\theta / 2)$ only to leading order since it can be shown that small-angle collisions dominate. We do, however, allow $q=2 x p / \alpha$ to be significant due to the large electron energies. Only the term including $\nu_{D}^{a b}$ in Eq. (1) then persists:

$$
\begin{gathered}
\nu_{D}^{e i}=\nu_{D, \mathrm{cs}}^{e i}\left(1+\frac{1}{Z_{\mathrm{eff}}} \sum_{j} \frac{n_{j}}{n_{e}} \frac{g_{j}(p)}{\ln \Lambda}\right), \\
g_{j}(p)=\int_{0}^{1} x^{-1}\left\{\left[Z_{j}-F_{j}(q)\right]^{2}-Z_{0, j}^{2}\right\} d x,
\end{gathered}
$$

where $\nu_{D, \mathrm{cs}}^{e i}=\tau_{c}^{-1} Z_{\mathrm{eff}} \gamma / p^{3}$ is the completely screened deflection frequency for superthermal particles, and $\tau_{c}=$ $\left(4 \pi n_{e} c r_{0}^{2} \ln \Lambda\right)^{-1}$ is the relativistic collision time. The effective charge is defined as $Z_{\text {eff }}=\sum_{j} n_{j} Z_{0, j}^{2} / n_{e}$, where $n_{e}$ represents the density of free electrons. The lower integration limit in $g_{j}(p)$ (which is formally $1 / \Lambda \ll 1$ ) has been set to zero, since the integrand is finite. We model the Coulomb logarithm according to $\ln \Lambda^{e e}=\ln \Lambda_{0}+$ $(1 / k) \ln \left\{1+\left[2(\gamma-1) / p_{T e}^{2}\right]^{k / 2}\right\}$ and $\ln \Lambda^{e i}=\ln \Lambda_{0}+(1 / k)$ $\ln \left[1+\left(2 p / p_{T e}\right)^{k}\right]$, where $p_{T e}$ is the thermal momentum. 
The parameter $k=5$ is chosen to give a smooth transition between the low-energy formula $\ln \Lambda_{0}=14.9-$ $0.5 \ln \left(n_{e}\left[10^{20} \mathrm{~m}^{-3}\right]\right)+\ln \left(T_{e}[\mathrm{keV}]\right) \quad$ [25] and the highenergy formula from Refs. [26,27].

To calculate the form factor, the electron charge density of the ion can be obtained via, e.g., DFT calculations; in this work we have used the numerical tool EXCITING [28]. Our calculations show that the form factor can be well described by a single-parameter model of the same form as that obtained from the Thomas-Fermi model by Kirillov et al. [16]: $F_{j, \mathrm{TF}-\mathrm{DFT}}(q)=N_{e, j} /\left[1+\left(q a_{j}\right)^{3 / 2}\right]$. This model, which we denote the Thomas-Fermi-DFT (TF-DFT) model, gives

$$
g_{j}(p)=\frac{2}{3}\left(Z_{j}^{2}-Z_{0, j}^{2}\right) \ln \left(y_{j}^{3 / 2}+1\right)-\frac{2}{3} \frac{N_{e, j}^{2} y_{j}^{3 / 2}}{y_{j}^{3 / 2}+1},
$$

where $y_{j}=2 a_{j} p / \alpha$. Note that the limit of complete screening $\left(g_{j}(p) \rightarrow 0\right)$ is reached as $p \rightarrow 0$ or for $Z_{j}=Z_{0, j}$. The parameter $a_{j}$-the effective ion size in units of Bohr radii-depends on the ion species and ionization degree, and was determined by fitting $g_{j}$ in Eq. (6) to Eq. (5) evaluated with the DFT output. For example, we obtain $a_{\mathrm{Ar}}=0.353$, $a_{\mathrm{Ar}^{+}}=0.329, a_{\mathrm{Ar}^{2+}}=0.306, a_{\mathrm{Ar}^{3+}}=0.283, a_{\mathrm{Ar}^{4+}}=0.260$, $a_{\mathrm{Ar}^{5+}}=0.238, a_{\mathrm{Xe}^{+}}=0.238$, and $a_{\mathrm{Be}^{+}}=0.414$.

The TF-DFT model agrees well with the prediction of full DFT simulations. Figure 1(a) shows the energydependent enhancement of the deflection frequency normalized to the completely screened value, together with the fit given in Eq. (6) for singly and doubly-ionized argon. The deflection frequency is already almost 2 orders of magnitude higher than the corresponding complete-screening value at electron energies of a few hundred $\operatorname{keV}(p \approx 1)$.

Inelastic collisions. - The energy loss in electronelectron collisions is described by the Bethe stoppingpower formula [15,29], which modifies the slowing-down frequency $\nu_{S}^{e e}$ describing collisional drag according to $\nu_{S}^{e e}=\nu_{S, \mathrm{cs}}^{e e}\left[1+\sum_{j}\left(n_{j} N_{e, j} / n_{e} \ln \Lambda\right)\left(\ln h_{j}-\beta^{2}\right)\right]$, where $\nu_{S, \mathrm{cs}}^{e e}=$ $\tau_{c}^{-1} \gamma^{2} / p^{3}$ is the completely screened, superthermal slowing-down frequency, $h_{j}=p \sqrt{\gamma-1} / I_{j}$, and $I_{j}$ is the mean excitation energy of the ion, normalized to the electron rest energy. In this work, the numerical values of $I_{j}$ for different ion species were obtained from Ref. [30]. This model is valid for $\gamma-1 \gg I_{j}$, corresponding to $p \gtrsim 0.03$ for both singly and doubly ionized argon. We provide here an interpolation formula, from matching the above to the lowenergy asymptote corresponding to complete screening, which we will refer to as the Bethe-like model:

$\nu_{S}^{e e}=\nu_{S, \mathrm{cs}}^{e e}\left[1+\sum_{j} \frac{n_{j} N_{e, j}}{n_{e} \ln \Lambda}\left(\frac{1}{k} \ln \left(1+h_{j}^{k}\right)-\beta^{2}\right)\right]$.

As in our model of $\ln \Lambda$, we set $k=5$.

Figure 1(b) shows the enhancement of the slowing-down frequency as a function of the electron energy. Note that already around a few tens of $\mathrm{keV}$, the enhancement using

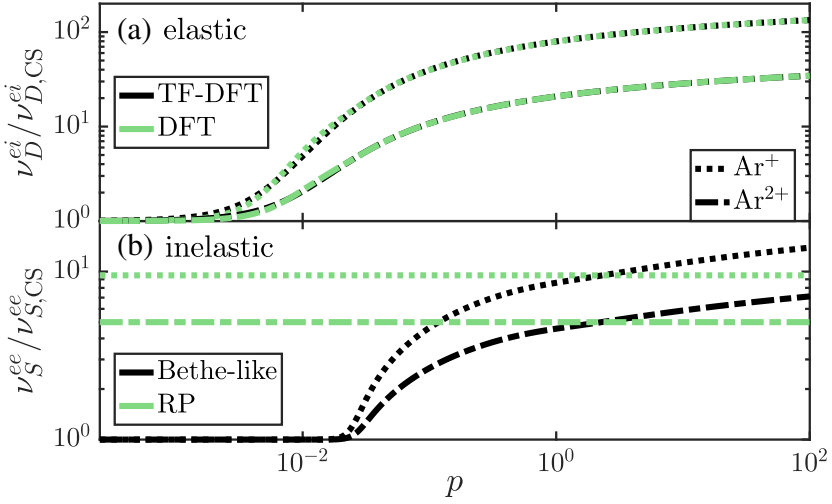

FIG. 1. (a) The deflection frequency and (b) the slowing-down frequency as a function of the incoming-electron momentum, normalized to the completely screened collision frequencies. The models employed here (TF-DFT and Bethe-like) are plotted in black, while the full DFT model and the approximate RP model are shown in green. Note in (a) the lines overlay almost exactly. A pure argon plasma [of either $\mathrm{Ar}^{+}$(dotted line) or $\mathrm{Ar}^{2+}$ (dashdotted line)], with $T=10 \mathrm{eV}$ and $n_{\mathrm{Ar}}=10^{20} \mathrm{~m}^{-3}$ was assumed, giving $\ln \Lambda_{0}^{+}=9.9$ and $\ln \Lambda_{0}^{2+}=10.3$.

the Bethe-like model (black) is an order of magnitude. The transition between the Bethe equation and the low-energy limit can be clearly seen at $p \approx 0.02$. It is instructive to compare these results to the RosenbluthPutvinski (RP) rule of thumb that the effect of inelastic collisions can be modeled by adding half of the bound electrons to the free electron density [31]: $\nu_{S, \mathrm{RP}}^{e e} \approx$ $\nu_{S, \mathrm{cs}}^{e e}\left[1+\frac{1}{2} \sum_{j} n_{j} N_{e, j} / n_{e}\right]$. This approximation [green line in Fig. 1(b)] leads to a much greater enhancement than the full formula up to $p \simeq 0.1$. This region in momentum space is important, since runaway generation is sensitive to the dynamics at the critical momentum $p_{c}$, which often is in the region $p_{c} \lesssim 0.1$. The effect of inelastic collisions on the electron-electron deflection frequency $\nu_{D}^{e e}$ does not follow from the stopping-power calculation, but is of order $X^{-1}$ smaller than $\nu_{D}^{e i}$ and can be ignored for low ionization degrees.

Numerical simulations. - The generalized collision operator presented here, consisting of Eqs. (1), (4), (6), and (7), has been implemented in the numerical tool CODE [32-34], which we use to solve the spatially homogeneous kinetic equation for electrons in 2D momentum space, including electric-field acceleration, collisions, and synchrotronradiation reaction losses.

We demonstrate the effects of reduced screening by investigating the decay phase of the runaway evolution. In the scenario considered, an electron distribution with an energetic runaway tail (with average energy $7.8 \mathrm{MeV}$ ), produced by a strong electric field, was used as the initial state. During the simulation of the decay phase, the weak electric field $E=2 E_{c}$ was used, which is well below the effective critical field if reduced screening effects are taken into account. To isolate the effect of reduced screening, 


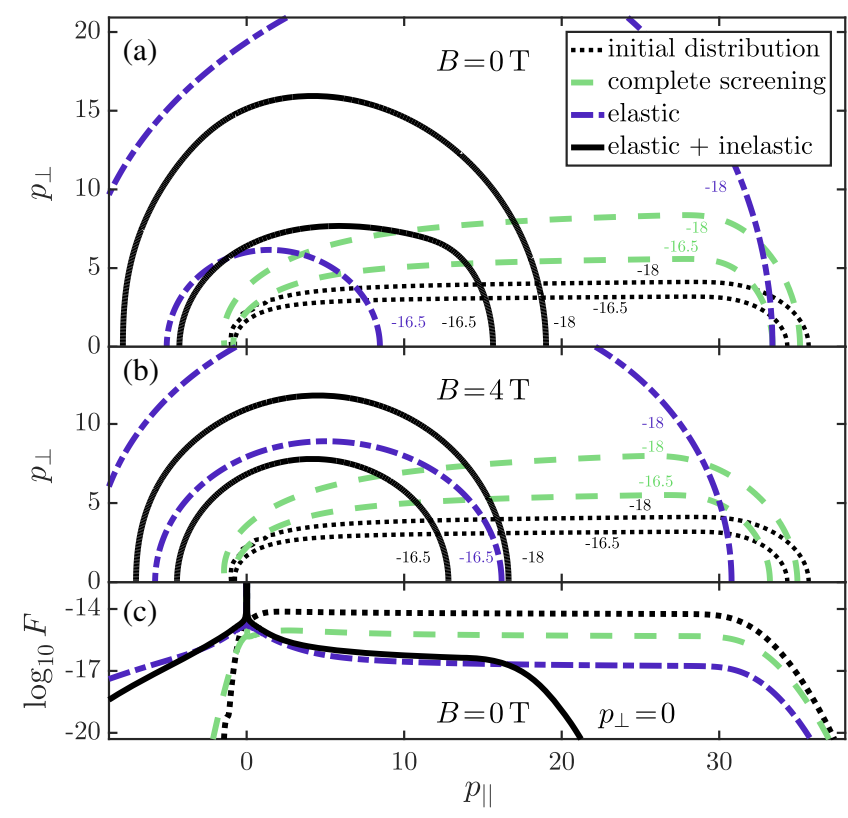

FIG. 2. Contours of the distribution after $25 \mathrm{~ms}$ of collisional deceleration from an initial beamlike state (dotted black line), with and without screening effects. The limit of complete screening for both $\nu_{D}^{e i}$ and $\nu_{S}^{e e}$ (dashed green line) is shown, together with the TF-DFT model for $\nu_{D}^{e i}$ but unmodified $\nu_{S}^{e e}$ (dash-dotted blue line), and with both the TF-DFT and Bethe-like models (solid black line). The contours $\log _{10}(F)=-16.5$ and -18 are shown, where $F=\left(2 \pi m_{e} T\right)^{3 / 2} f_{e} / n_{e}$, with (a) $B=0 \mathrm{~T}$ and (b) $B=4 \mathrm{~T}$. A parallel cut through the distribution of (a) is shown in (c). Parameters: $T=10 \mathrm{eV}, n_{\mathrm{H}}=10^{20} \mathrm{~m}^{-3}$ and $\mathrm{Ar}^{+}$ with density $n_{\mathrm{Ar}}=n_{\mathrm{H}}, E=2 E_{c}$.

avalanche runaway generation (which is unimportant on the simulation time scale [31]) was neglected, and bremsstrahlung (which can sometimes be an important energy-loss mechanism $[35,36])$ does not affect the dynamics significantly at the energies considered.

The effect of reduced screening on the electron distribution is shown in Fig. 2(a) without and 2(b) with synchrotron radiation, in a plasma with equal amounts of singly ionized argon and hydrogen. As shown in the figure, the enhanced deflection frequency (dash-dotted blue line) acts to make the distribution function isotropic, as can be seen by a comparison to the completely screened case (dashed green line). Energy losses are induced when, in addition, the slowing-down model of inelastic collisions with bound electrons is included (solid black line). The synergy with synchrotron radiation further enhances the energy loss as shown in Fig. 2(b). The one-dimensional plot in Fig. 2(c) offers a complementary view of the distribution function; note that the maximum runaway energy is lower if inelastic collisions are included, compared to only considering elastic collisions.

The changes to the distribution function presented in Fig. 2 have significant implications for the decay of a runaway-electron current, as shown in Fig. 3. Since the decay time to a good approximation is proportional to

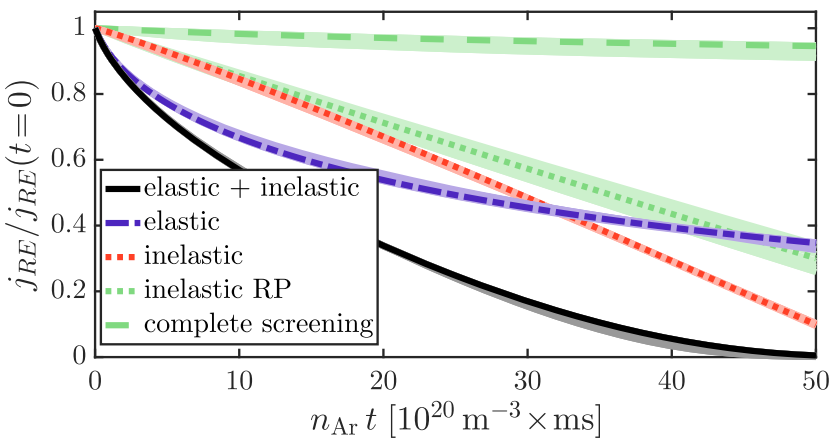

FIG. 3. Decay of the runaway-electron current as a function of argon density and time. The full model, with both the TF-DFT and Bethe-like contributions (solid black line), as well as the $\nu_{D}^{e i}$ (dash-dotted blue line) and $\nu_{S}^{e e}$ (dotted red line) models separately, are shown. These are compared to the RP model for inelastic collisions (dotted green line) and the limit of complete screening (dashed green line). The initial distribution of Fig. 2 was used, assuming a $\mathrm{H}$ plasma with $n_{\mathrm{H}}=10^{20} \mathrm{~m}^{-3}$, and $\mathrm{Ar}^{+}$ impurities with $n_{\mathrm{Ar}}=10 n_{\mathrm{H}}$; the light bands show the range of results obtained by varying the argon density such that $n_{\mathrm{Ar}} \in\left[0.5 n_{\mathrm{H}}, 100 n_{\mathrm{H}}\right] . T=10 \mathrm{eV}, E=2 E_{c}$, and $B=2 \mathrm{~T}$.

$1 / n_{\mathrm{Ar}}$ for $n_{\mathrm{Ar}} \gtrsim n_{\mathrm{H}}$ (i.e., when $\nu_{D}^{e i} \gg \nu_{D, \mathrm{cs}}^{e i}$ and $\nu_{S}^{e e} \gg \nu_{S, \mathrm{cs}}^{e e}$ ), the runaway-electron current is shown as a function of $n_{\mathrm{Ar}} t$. With the full model of reduced screening, the current-decay time is reduced by orders of magnitude compared to the complete-screening model. The effect on the current is due to the combination of the inelastic and the elastic collisions: elastic collisions are most important when the distribution is narrow in the pitch angle, while inelastic collisions will dominate at later times, when the distribution is sufficiently isotropic so that elastic scattering is less efficient. The RP model (which was applied for momenta $p>10 p_{T e}$ ) underestimates the decay rate resulting from inelastic collisions, and shows a widely different current evolution compared to the full model.

The bands in Fig. 3 represent an impurity density scan over 2 orders of magnitude, showing only very slight variation from the linear relationship between the decay rate and $n_{\mathrm{Ar}}$. Similarly, the current decay is insensitive to the electric field as long as it is significantly lower than the effective critical field. Although the relative importance of elastic and inelastic collisions is influenced by the width of the distribution function in the pitch angle, the overall effect on the current decay is not strongly affected as long as the initial distribution is forward beamed.

While the current decay shown in Fig. 3 is a robust result when the electric field is constant, it neglects the inductive coupling between the current, $I$, and the electric field in a tokamak, which is allowed when $I \lesssim 250 \mathrm{kA}$ [37]. The opposite, highly inductive limit leads to a current decay rate proportional to the critical electric field [37]: $d I / d t=2 \pi R E_{c}^{\mathrm{eff}} / L$, where $L \sim \mu_{0} R$ is the self-inductance and $R$ is the major radius. To calculate $E_{c}^{\mathrm{eff}}$, which is increased due to reduced screening compared to the classical value $E_{c}$, we assume fast pitch-angle dynamics 
following the procedure in Refs. [5,38]. For a $\mathrm{H}$ plasma with up to triply ionized Ar impurities and densities in the range $n_{\mathrm{Ar}} \gtrsim 0.1 n_{\mathrm{H}}$, and neglecting synchrotron losses $\left(B[\mathrm{~T}]^{2} \lesssim n_{\mathrm{Ar}}\left[10^{18} \mathrm{~m}^{-3}\right]\right)$, we obtain

$$
\frac{E_{c}^{\mathrm{eff}}}{E_{c}} \approx 1+\frac{1}{\ln \Lambda_{0}}\left(7-\ln \sqrt{T_{\mathrm{eV}}}+240 \frac{n_{\mathrm{Ar}, \mathrm{tot}}}{n_{e}}\right) .
$$

This large enhancement of the critical electric field has significant contributions from elastic and inelastic collisions, implying that both effects are important for the runaway dynamics regardless of the inductance.

Conclusions. - In this Letter, we give convenient analytical expressions for the effect of reduced screening on the collisional deflection and slowing-down frequencies, derived from first principles. The model is formally correct where the Born approximation is valid $(v / c \gg Z \alpha)$, but is applicable for all electron energies due to a matching to the completely screened low-energy limit. For the first time, we investigate the electron dynamics using kinetic simulations, and find that the reduced-screening effect of bound electrons has a large impact on the distribution function of runaway electrons. The enhancement of both collisional drag and pitch-angle scattering lead to significant energy loss, the latter due to the increased synchrotron radiation. We provide a formula for the effective critical field-the threshold for runaway generation-which can be used to predict the runaway-current decay time in tokamaks. Our results indicate that runaway beams will be strongly damped even in the presence of weakly ionized impurities, in agreement with experiments [10]. Given the impact of collisions with screened nuclei on the dynamics of runaway electrons, the effects detailed here should be considered in all situations where fast electrons interact with partially ionized impurities, e.g., lightning discharges, tokamak disruptions, and laser-plasma interaction.

This work was supported by the Swedish Research Council (Dnr. 2014-5510), the Knut and Alice Wallenberg Foundation, and the European Research Council (ERC-2014-CoG Grant No. 647121). The authors are grateful for stimulating discussions with Matt Landreman, George Wilkie, and Christian Forssén.

*hesslow@chalmers.se

[1] H. Dreicer, Phys. Rev. 115, 238 (1959).

[2] J. Connor and R. Hastie, Nucl. Fusion 15, 415 (1975).

[3] G. D. Holman, Astrophys. J. 293, 584 (1985).

[4] J. R. Dwyer, Phys. Plasmas 14, 042901 (2007).

[5] N. G. Lehtinen, T. F. Bell, and U. S. Inan, J. Geophys. Res: Space Physics 104, 24699 (1999).

[6] P. B. Parks and T.E. Cowan, Phys. Plasmas 14, 013102 (2007).

[7] P. Helander, L.-G. Eriksson, and F. Andersson, Plasma Phys. Controlled Fusion 44, B247 (2002).

[8] C. Reux et al., Nucl. Fusion 55, 093013 (2015).

[9] M. Shimada et al., Nucl. Fusion 47, S1 (2007).
[10] E. M. Hollmann, P. B. Parks, N. Commaux, N. W. Eidietis, R. A. Moyer, D. Shiraki, M. E. Austin, C. J. Lasnier, C. Paz-Soldan, and D. L. Rudakov, Phys. Plasmas 22, 056108 (2015).

[11] A. H. Boozer, Phys. Plasmas 22, 032504 (2015).

[12] M. Lehnen et al., J. Nucl. Mater. 463, 39 (2015).

[13] E. Hollmann, M. Austin, J. Boedo, N. Brooks, N. Commaux, N. Eidietis, D. Humphreys, V. Izzo, A. James, T. Jernigan, A. Loarte, J. Martin-Solis, R. Moyer, J. Munoz-Burgos, P. Parks, D. Rudakov, E. Strait, C. Tsui, M. V. Zeeland, J. Wesley, and J. Yu, Nucl. Fusion 53, 083004 (2013).

[14] L. D. Landau and E. M. Lifshitz, Quantum Mechanics: Nonrelativistic Theory (Elsevier, New York, 2013), Vol. 3.

[15] H. Bethe, Ann. Phys. (Berlin) 397, 325 (1930).

[16] V. D. Kirillov, B. A. Trubnikov, and S. A. Trushin, Fiz. Plazmy 1, 218 (1975).

[17] V. Zhogolev and S. Konovalov, Characteristics of interaction of energetic electrons with heavy impurity ions in a tokamak plasma, VANT or Problems of Atomic Sci. and Tech. series Thermonuclear Fusion 37, 71 (2014) (in Russian).

[18] D. Mosher, Phys. Fluids 18, 846 (1975).

[19] J. R. Martin-Solis, A. Loarte, and M. Lehnen, Phys. Plasmas 22, 092512 (2015).

[20] Y. L. Igitkhanov, Contrib. Plasma Phys. 52, 460 (2012).

[21] M. N. Rosenbluth, W. M. MacDonald, and D. L. Judd, Phys. Rev. 107, 1 (1957).

[22] H. Akama, J. Phys. Soc. Jpn. 28, 478 (1970).

[23] P. Helander and D. Sigmar, Collisional Transport in Magnetized Plasmas (Cambridge University Press, Cambridge, England, 2005).

[24] W. Heitler, The Quantum Theory of Radiation (Dover Publications, Inc, New York, 1954) Vol. 86.

[25] J. Wesson, Tokamaks, 4th ed. (Oxford University Press, New York, 2011).

[26] R. Gould, Physica (Utrecht) 60, 145 (1972).

[27] A. A. Solodov and R. Betti, Phys. Plasmas 15, 042707 (2008).

[28] A. Gulans, S. Kontur, C. Meisenbichler, D. Nabok, P. Pavone, S. Rigamonti, S. Sagmeister, U. Werner, and C. Draxl, J. Phys. Condens. Matter 26, 363202 (2014).

[29] J. D. Jackson, Classical Electrodynamics (Wiley, New York, 1999).

[30] S. P. Sauer, J. Oddershede, and J. R. Sabin, in Concepts of Mathematical Physics in Chemistry: A Tribute to Frank E. Harris-Part A, Advances in Quantum Chemistry Vol. 71 (Academic Press, New York, 2015), p. 29.

[31] M. Rosenbluth and S. Putvinski, Nucl. Fusion 37, 1355 (1997).

[32] M. Landreman, A. Stahl, and T. Fülöp, Comput. Phys. Commun. 185, 847 (2014).

[33] A. Stahl, E. Hirvijoki, J. Decker, O. Embréus, and T. Fülöp, Phys. Rev. Lett. 114, 115002 (2015).

[34] A. Stahl, O. Embréus, G. Papp, M. Landreman, and T. Fülöp, Nucl. Fusion 56, 112009 (2016).

[35] M. Bakhtiari, G. J. Kramer, M. Takechi, H. Tamai, Y. Miura, Y. Kusama, and Y. Kamada, Phys. Rev. Lett. 94, 215003 (2005).

[36] O. Embréus, A. Stahl, and T. Fülöp, New J. Phys. 18, 093023 (2016).

[37] B. N. Breizman, Nucl. Fusion 54, 072002 (2014).

[38] P. Aleynikov and B. N. Breizman, Phys. Rev. Lett. 114, 155001 (2015). 